\title{
Reconstructing phylogenies from noisy quartets in polynomial time with a high success probability
} Gang Wu*1, Ming-Yang Kao*2, Guohui Lin¹ and Jia-Huai You ${ }^{1}$

\author{
Address: ${ }^{1}$ Department of Computing Science, University of Alberta, Edmonton, Alberta T6G 2E8, Canada and ${ }^{2}$ Department of Electrical \\ Engineering and Computer Science, Northwestern University, Evanston, IL 60208, USA \\ Email: Gang Wu* - wgang@cs.ualberta.ca; Ming-Yang Kao* - kao@cs.northwestern.edu; Guohui Lin - ghlin@cs.ualberta.ca; Jia- \\ Huai You - you@cs.ualberta.ca \\ * Corresponding authors
}

Published: 24 January 2008

Algorithms for Molecular Biology 2008, 3:I doi:10.1 186/1748-7। I88-3-I

This article is available from: http://www.almob.org/content/3/I/I

(c) 2008 Wu et al; licensee BioMed Central Ltd.

This is an Open Access article distributed under the terms of the Creative Commons Attribution License (http://creativecommons.org/licenses/by/2.0), which permits unrestricted use, distribution, and reproduction in any medium, provided the original work is properly cited.
Received: 14 November 2006

Accepted: 24 January 2008

\begin{abstract}
Background: In recent years, quartet-based phylogeny reconstruction methods have received considerable attentions in the computational biology community. Traditionally, the accuracy of a phylogeny reconstruction method is measured by simulations on synthetic datasets with known "true" phylogenies, while little theoretical analysis has been done. In this paper, we present a new model-based approach to measuring the accuracy of a quartet-based phylogeny reconstruction method. Under this model, we propose three efficient algorithms to reconstruct the "true" phylogeny with a high success probability.

Results: The first algorithm can reconstruct the "true" phylogeny from the input quartet topology set without quartet errors in $O\left(n^{2}\right)$ time by querying at most $(n-4) \log (n-1)$ quartet topologies, where $n$ is the number of the taxa. When the input quartet topology set contains errors, the second algorithm can reconstruct the "true" phylogeny with a probability approximately I - $p$ in $O\left(n^{4} \log n\right)$ time, where $p$ is the probability for a quartet topology being an error. This probability is improved by the third algorithm to approximately $\frac{1}{1+q^{2}+\frac{1}{2} q^{4}+\frac{1}{16} q^{5}}$, where $q=\frac{p}{1-p}$, with running time of $O\left(n^{5}\right)$, which is at least 0.984 when $p<0.05$.

Conclusion: The three proposed algorithms are mathematically guaranteed to reconstruct the "true" phylogeny with a high success probability. The experimental results showed that the third algorithm produced phylogenies with a higher probability than its aforementioned theoretical lower bound and outperformed some existing phylogeny reconstruction methods in both speed and accuracy.
\end{abstract}

\section{Background}

Evolution is a basic process in biology. The evolutionary history, referred to as phylogeny, of a set of taxa can be mathematically defined as a tree where the leaves are labeled with the given taxa and the internal nodes represent extinct or hypothesized ancestors. There are rooted and unrooted phylogenies. In a rooted phylogeny, an edge specifies the parent-child relationship and the root repre- 
sents a common ancestor of all the taxa. A rooted phylogeny is called binary or resolved if every internal node has exactly two children. In an unrooted phylogeny, there is no parent-child relationship specified for an edge; and it is called binary or resolved if every internal node has degree exactly 3 .

There have been many works on how to reconstruct rooted and unrooted phylogenies [1-3]. It is already known that rooted phylogenies and unrooted phylogenies can be transformed into each other [4], for example, by using an outgroup. In the remainder of this paper, a phylogeny refers to an unrooted binary phylogeny unless explicitly specified otherwise.

Given a taxon set $S$, each subset of four taxa of $S$ is called a quartet of $S$. In recent years, quartet-based phylogeny reconstruction methods have received considerable attentions in the computational biology community. In comparison with other phylogeny reconstruction methods, an advantage of quartet-based methods is that they can overcome the data disparity problem [5]. An unrooted phylogeny (or topology) of a quartet is called its quartet topology. Given a quartet $\left\{s_{1}, s_{2}, s_{3}, s_{4}\right\}$ of $S$, there are three possible topologies associated with it, up to symmetry. These three quartet topologies are shown in Figure 1. For simplicity, we use $\left[s_{1}, s_{2} \mid s_{3}, s_{4}\right]$ to denote the quartet topology in which the path connecting $s_{1}$ and $s_{2}$ does not intersect the path connecting $s_{3}$ and $s_{4}$ (see Figure 1(a)). The other two quartet topologies are $\left[s_{1}, s_{3} \mid s_{2}, s_{4}\right]$ and $\left[s_{1}, s_{4} \mid s_{2}, s_{3}\right]$.

Given a taxon set $S$ and a phylogeny $T$ on $S$, we can see that trimming all the other nodes (including the root if $T$ is rooted) from $T$ gives exactly one topology for every quartet of $S$. The quartet-based phylogeny reconstruction works inversely to first build a phylogeny for every quartet and then infer an overall phylogeny for the whole set of taxa. Suppose that $Q$ is the set of quartet topologies built in the first step of a quartet-based phylogeny reconstruction, which can be done by various quartet inference methods [6-8]. If there exists a phylogeny $T$ such that a quartet topology $q$ in $Q$ is the same as the one derived from $T$, then we say that $T$ satisfies $q$, and $q$ is consistent with

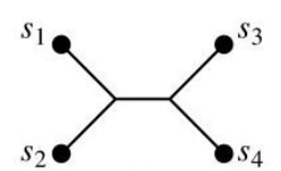

(a)

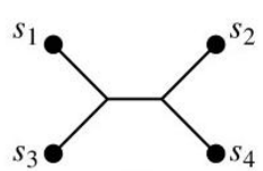

(b)

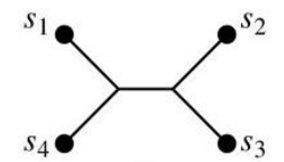

(c)

\section{Figure I}

The three possible quartet topologies for quartet $\left\{s_{1}, s_{2}, s_{3}\right.$, $\left.s_{4}\right\}$.
$T$. If there exists a phylogeny $T$ satisfying all quartet topologies in $Q$, then we say that $Q$ is compatible and $T$ is the (unique) phylogeny associated with $Q$. In the ideal case where all quartet topologies are "correct," i.e., $Q$ is compatible, the task of assembling an overall phylogeny is easy and can be done in $O\left(n^{4}\right)$ time [9], where $n$ is the number of taxa under consideration. In practice, however, some quartet topologies may be erroneous. Therefore, the set of quartet topologies may contain conflicting quartet topologies. This possibility complicates the overall quartet-based phylogeny reconstruction and presents an interesting computational challenge.

Given a taxon set $S$, we define the phylogeny that reveals the correct relationships among the taxa in $S$ as the "true" phylogeny on $S$, denoted as $T_{\text {true. }}$. The accuracy of a phylogeny reconstruction method is the extent to which the generated phylogeny agrees with the "true" phylogeny. In many applications, the "true" phylogeny is not available to us for real-life instances in the study of evolution. Therefore, to investigate the accuracy of different reconstruction methods, synthetic data are created with simulations using a given evolutionary model, where the "true" phylogeny is known. If a quartet topology $q \in Q$ conflicts with $T_{\text {true }^{\prime}}$ then $q$ is a quartet error. Given a quartet topology set containing possible quartet errors, current phylogeny reconstruction methods seek to estimate the "true" phylogeny in one of the following two ways: (1) by a specific algorithm that leads to the determination of a phylogeny; or (2) by defining a measurement for the quality of generated phylogenies and searching for an optimal phylogeny. Purely algorithmic methods in the first category integrate phylogeny reconstruction and the definition of the preferred phylogeny tightly. These methods include quartet puzzling [10], the short quartet method [8], and semi-definite programming [4]. The methods in the first category tend to be computationally fast because they proceed directly toward the final solution without the evaluation of a large number of competing phylogenies. However, they can achieve high accuracy only on some specific datasets. Other statistical methods such as bootstrapping [11] are incorporated to assess the confidence of a found phylogeny, which requires extra computational time but may generate better phylogenies. These statistical methods have their limitations and may fail in some situations [12].

The second category of methods first define a score for each given quartet topology and then use combinatorial algorithms to find a phylogeny that achieves the optimal score. For example, the Maximum Quartet Consistency (MQC) problem [13], which is NP-hard, aims to compute a phylogeny which respects as many quartet topologies as possible. Several attempts have been made to solve MQC optimally $[5,14,15]$ or approximately $[16,17]$. The hyper- 
cleaning algorithm proposed in [18] aims to reconstruct a phylogeny that minimizes a certain quartet distance value for measuring the quartet errors. The complexity of the hypercleaning algorithm is $O\left(n^{5} f(2 m)+n^{7} f(m)\right)$, where $f(m)=4 m^{2}(1+2 m)^{4 m}, n$ is the number of taxa, and $m$ is a value based on the quartet distance model. These methods tend to be much slower than those in the first category but have higher accuracy. For datasets with a relatively large number of quartet errors, the optimal phylogenies produced by these methods may not be unique, and one must provide additional measurements to estimate the "true" phylogeny.

Traditionally, the performance accuracy of a phylogeny reconstruction method is measured by simulations on synthetic datasets with a known "true" phylogeny, while little theoretical analysis has been done. In this paper, we propose a new model-based approach to measuring the accuracy of a quartet-based phylogeny reconstruction method, i.e., to analyze the probability of reconstructing the "true" phylogeny.

\section{Methods}

We define our data model and describe our three phylogeny reconstruction algorithms in this section.

\section{Probabilistic model of quartet generation}

In this section, we define a probabilistic model for the quartet-based phylogeny reconstruction and introduce some terminologies that will be used in the discussion of three new algorithms.

Given a quartet topology set $Q$ on a taxon set $S=\left\{s_{1}\right.$, $\left.s_{2}, \ldots, s_{n}\right\}, Q$ is complete if $Q$ contains exactly one quartet topology for every quartet of $S$. In this paper, we assume $Q$ is complete. Given a phylogeny $T$ on a taxon set $S=\left\{s_{1}\right.$, $\left.s_{2}, \ldots, s_{n}\right\}, n$ is the size of $T$, and we use $Q_{T}$ to denote the complete quartet topology set induced by $T$. Given $T_{\text {true' }}$ our simulation model first generates a complete quartet topology set $Q_{T_{\text {true }}}$ for $T_{\text {true. }}$. For every quartet topology in $Q_{T_{\text {true }}}$, with probability 1 - $p(0 \leq p \leq 1)$ our simulation model does not do anything to it, and with probability $\frac{p}{2}$ changes its topology into each of the other two topologies. In this way, the model generates the input quartet topology set $Q$, and consequently every quartet topology in the generated set $Q$ has the same probability $p$ of being a quartet error. This probability $p$ is called the quartet error probability associated with the instance. Under this model, our main computational objective is to reconstruct $T_{\text {true }}$ from $Q$ with a high success probability while minimizing the time complexity.

In practice, the quartet error probability $p$ mainly depends on the quality of the quartet inference methods, such as the Four-point method [9], the Neighbor Joining method [6], and the Ordinal Quartet method [7]. Simulation results in [7] show that the Ordinal Quartet method can achieve over $80 \%$ accuracy while inferring quartet topologies. Therefore, in our model we assume that current quartet inference methods can infer more correct quartet topologies than erroneous ones. In particular, we assume the quartet error probability $0 \leq p<\frac{1}{3}$. As this paper focuses on phylogeny reconstruction, we also assume that the time complexity of inferring one quartet topology is $O(1)$.

\section{An $O\left(n^{2}\right)$-time algorithm for reconstructing $T_{\text {true }}$ when $p=$ 0}

In this section, we assume that no quartet errors exist in $Q$. Our algorithm is based on the following classic result by Jordan [19].

Lemma 1 (see [19]) Given a tree T with $n$ leaves, there exists an internal node whose removal partitions the tree into connected components, each with at most $\frac{n}{2}$ leaves, and such a node can be found in linear time.

Given an unrooted binary phylogeny $T$, if we remove an internal node $v$ from $T, T$ will be divided into three subphylogenies. We denote these three sub-phylogenies as $T$ - $\{v\}$. Based on Lemma 1, there exists an internal node $v$ in $T$ such that each of the trees in $T-\{v\}$ has at most $\frac{n}{2}$ leaves. An internal node $v$ of $T$ having such a property is called a separator of $T$. Notice that a phylogeny $T$ may have more than one separator, but our algorithms in Tables 1 , 2 , and 3 need only one of them. Given a phylogeny $T$ and a separator $v$ of $T$, we can merge two sub-phylogenies of $T$ - $\{v\}$ into one leaf node (replacing the separator $v$ ), which is treated as a super taxon to represent the union of the taxon sets of the two merged sub-phylogenies.

Given a quartet topology set $\mathrm{Q}$ with no quartet errors, we can start with a randomly selected quartet topology q, which forms an initial phylogeny T4 on 4 taxa, and then iteratively insert a new taxon to grow the phylogeny. To ensure that the true phylogeny on the whole taxon set is recovered, in the i-th iteration to insert taxon si+4, we first locate a separator, $v$, of phylogeny $\mathrm{Ti}+3$. Then, we ran- 
Table I:

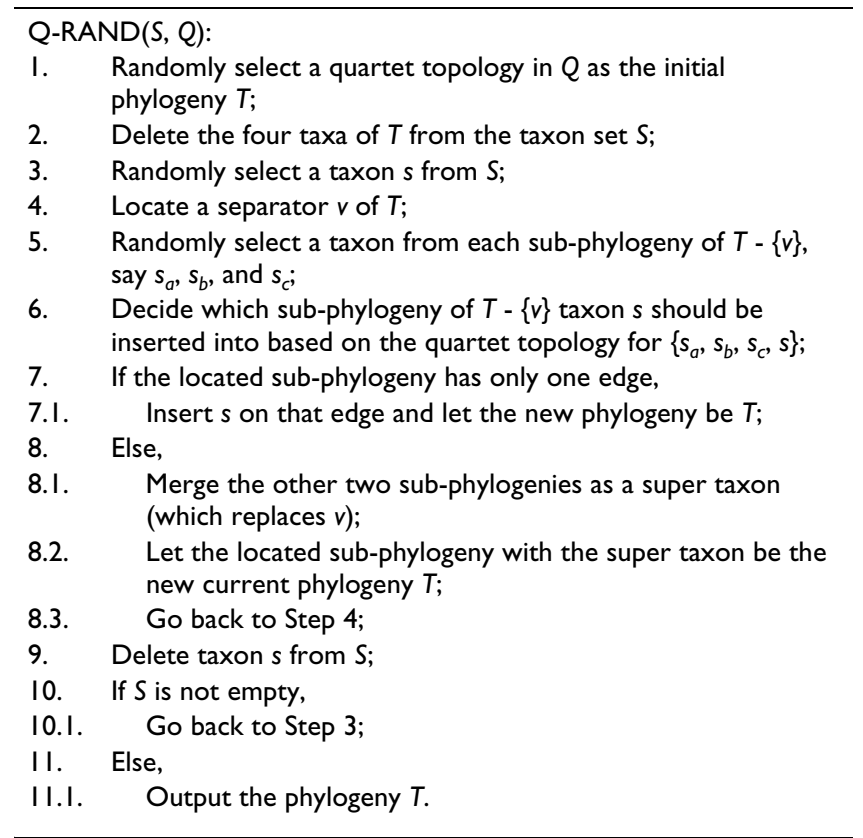

domly select a taxon from each of the three sub-phylogenies of $\mathrm{Ti}+3-\{\mathrm{v}\}$. Suppose that these three selected taxa are $\mathrm{sa}, \mathrm{sb}$, and sc. We proceed to check the given topology in $\mathrm{Q}$ on quartet $\{\mathrm{sa}, \mathrm{sb}, \mathrm{sc}, \mathrm{si}+4\}$. Based on that topology, we can determine which sub-phylogeny taxon si+4 should be inserted into. For example, if the topology is $[\mathrm{sa}, \mathrm{sb} \mid \mathrm{sc}$, si+4], then si+4 should be inserted into the sub-phylogeny that contains scas its leaf. Recursively, we treat the other two sub-phylogenies as a super taxon (which replaces the

\section{Table 2:}

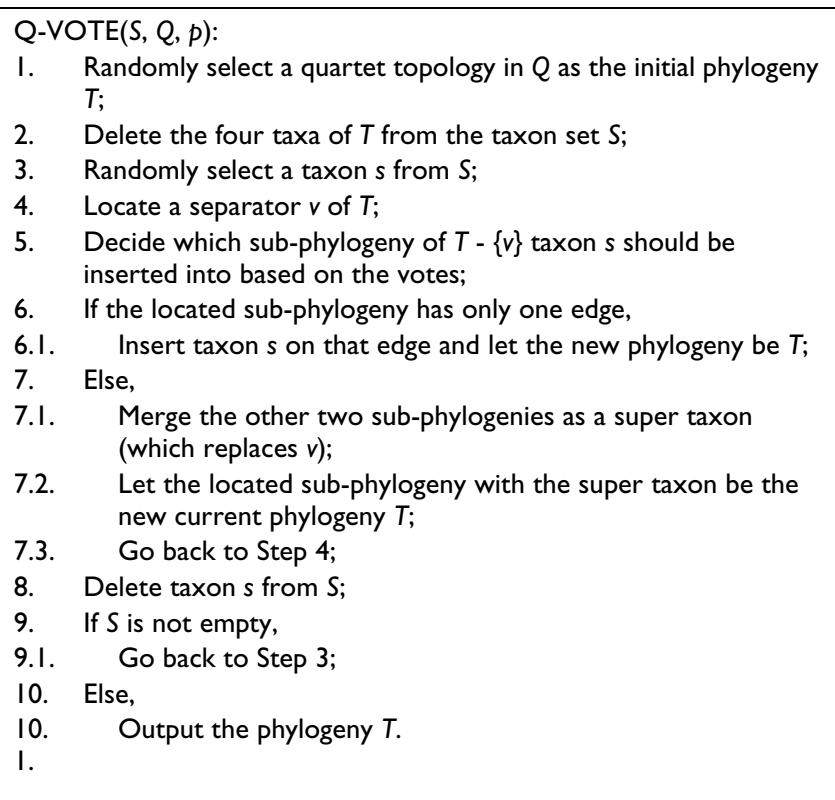

Table 3:

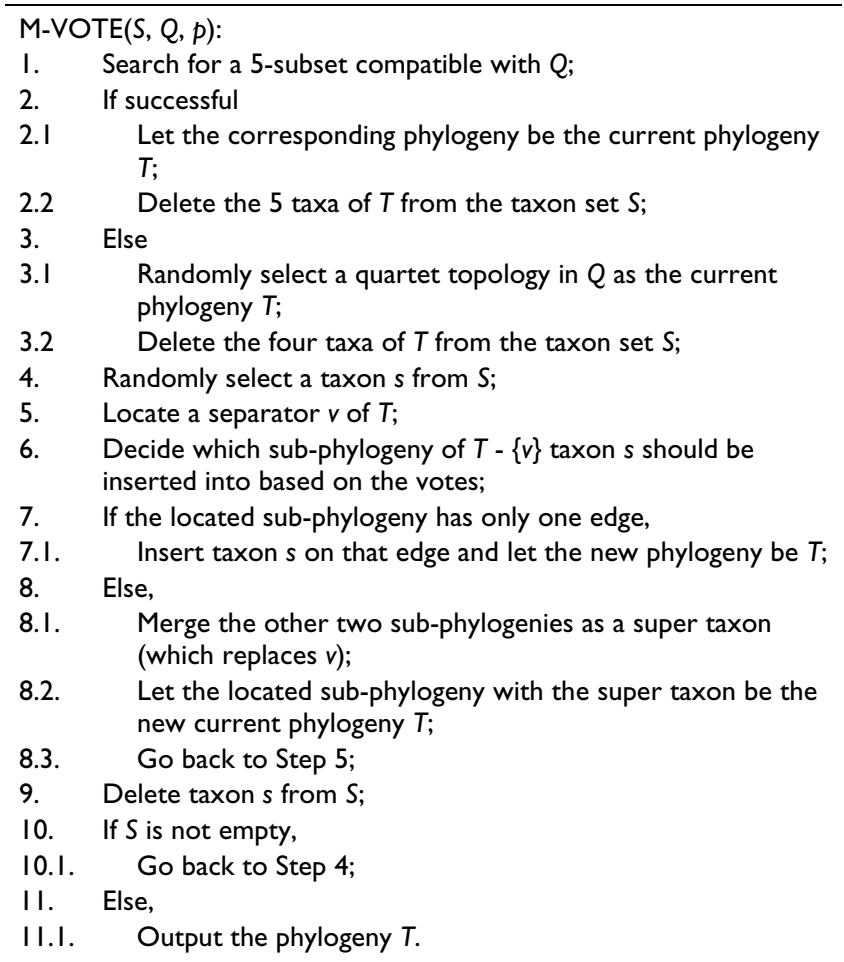

separator $\mathrm{v}$ ) on the located sub-phylogeny to generate a new phylogeny, and to determine the location in this new phylogeny where taxon si+4 should be inserted. A highlevel description of this algorithm Q-RAND is summarized in Table 1.

Theorem 2 Given a quartet topology set $Q$ with no quartet errors, $T_{\text {true }}$ can be constructed in $O\left(n^{2}\right)$ time by querying at most $(n-4) \log (n-1)$ quartet topologies in $Q$.

PROOF. The Q-RAND algorithm described above and detailed in Table 1 can be employed to construct the true phylogeny, where one can easily see that the final phylogeny obtained after inserting all the taxa satisfies all the quartet topologies in $Q$, and therefore it is $T_{\text {true }}$.

In the $i$-th iteration, Q-RAND needs to query at most $\log (i$ +3 ) quartet topologies. Therefore, the total number of quartet topologies need to be queried is at most $\log 4+\log$ $5+\cup+\log (n-1) \leq(n-4) \log (n-1)$. As we only need $O(1)$ time to infer each queried quartet topology, the time complexity of querying these quartet topologies is $O(n \log n)$.

Based on Lemma 1, finding a separator of phylogeny $T_{i}$ takes $O(i)$ time. Thus the time of finding the separators during the $i$-th iteration is $O(i+i / 2+U+1)=O(i)$. The overall time of Q-RAND is therefore $O\left(n^{2}\right)$. 
An experiment is a rooted phylogeny on three taxa. There has been extensive work on reconstructing phylogenies from a set of experiments with no errors. In general, there is a trade-off between the number of queried experiments and the running time. Kannan et al. [20] gave an $\Omega(n \log$ $n$ ) lower bound of queried experiments for reconstructing rooted binary phylogenies in $O\left(n^{2}\right)$ time. Kao et al. [21] presented a randomized algorithm with running time $O(n$ $\log n \log \log n)$ using $O(n \log n \log \log n)$ experiments. The fastest algorithm [22] so far is a deterministic algorithm which can reconstruct the true phylogeny in $O(n \log$ $n)$ time by querying at most $n(\log n+O(1))$ experiments. Although these algorithms and complexity results are for reconstructing phylogenies from experiments, they also apply to quartet-based phylogeny reconstruction through straightforward transformation. Therefore, algorithm QRAND achieves the lower bound of queried quartet topologies for phylogeny reconstruction from a given quartet topology set without errors. Q-RAND will be the base structure of our algorithms for the case with quartet errors.

\section{Reconstructing $\mathbf{T}_{\text {true }}$ with a high success probability when 0 $<\mathrm{p}<\frac{1}{3}$}

If the input quartet topology set $Q$ contains quartet errors, then algorithm Q-RAND may make a wrong decision while locating the sub-phylogeny where taxon $s_{i}$ should be inserted. In this section, we address this issue by adding a voting scheme to algorithm Q-RAND to aggregate the information in the correct quartet topologies. The key observation is that, when $p$ is small, in order to incorrectly identify the location for a new taxon, there must exist many quartet errors among the queried quartet topologies that all support the decision, which however is unlikely.

The new algorithm is called Q-VOTE, which also starts with an randomly picked quartet topology. In the $i$-th iteration to insert taxon $s_{i+4}$, the algorithm first locates a separator, $v$, of phylogeny $T_{i+3}$. It then queries all the possible quartet topologies on $\left\{s_{a^{\prime}} s_{b^{\prime}} s_{c^{\prime}} s_{i+4}\right\}$, where $s_{a^{\prime}} s_{b^{\prime}}$ and $s_{c}$ come from the taxon sets of the three sub-phylogenies of $T_{i+3}-\{v\}$, respectively. If a sub-phylogeny contains a super taxon, which is formed by merging two sub-phylogenies in a previous step, all the taxa represented by that super taxon are also taken into consideration. Suppose that the taxon sets of the three sub-phylogenies have sizes $m_{1}, m_{2}$, and $m_{3}$, respectively. Then there are $m_{1} \times m_{2} \times m_{3}$ quartet topologies that we need to consider. Each quartet topology gives a vote for a sub-phylogeny into which taxon $s_{i+4}$ should be inserted. For example, the quartet topology $\left[s_{a^{\prime}}\right.$ $\left.s_{b} \mid s_{c^{\prime}} s_{i+4}\right]$ gives a vote on the sub-phylogeny whose taxon set includes $s_{c}$. The algorithm then chooses the sub-phylogeny that has the maximum votes and recursively calls the above procedure until the location of taxon $s_{i+4}$ is determined. We call each recursive step described above a decision to locate taxon $s_{i+4}$. In each decision, the algorithm needs to query $O\left(i^{3}\right)$ quartet topologies, and $\log i$ decisions are needed to determine the final location of taxon $s_{i+4}$. Therefore, the overall running time of algorithm Q-VOTE is $O\left(n^{4} \log n\right)$. A high-level description of algorithm Q-VOTE is summarized in Table 2.

Theorem 3 When $0<p<\frac{1}{3}$, algorithm Q-VOTE can reconstruct $T_{\text {true }}$ in $O\left(n^{4} \log n\right)$ time with a probability at least $(1-p) \prod_{j=4}^{n-1}\left[1-\sum_{k=\frac{j-2}{2}}^{j-2}\left(\begin{array}{c}j-2 \\ k\end{array}\right) p^{k}(1-p)^{j-2-k}\right]^{\log j}$,

where $n$ is the size of the input taxon set and $p$ is the quartet error probability of the input quartet topology set.

PROOF. Suppose that the algorithm queries $N$ quartet topologies when it makes one decision of locating taxon $s_{j+1}$ on a phylogeny $T_{j}$ with $j$ taxa. It is easy to see that $N \geq$ $j-2$. The algorithm makes a wrong decision only if the number of quartet errors among these queried quartet topologies is at least $\frac{N}{2}$. (Note that, however, the existence of at least $\frac{N}{2}$ quartet errors does not necessarily imply the misplacement of taxon $s_{j+1}$.) We know that each quartet topology has a probability $p$ to be a quartet error. Therefore, the number of quartet errors follows a binomial distribution, and the probability that the algorithm makes a wrong decision is at most

$$
\sum_{k=\frac{N}{2}}^{N}\left(\begin{array}{c}
N \\
k
\end{array}\right) p^{k}(1-p)^{N-k} \leq \sum_{k=\frac{j-2}{2}}^{j-2}\left(\begin{array}{c}
j-2 \\
k
\end{array}\right) p^{k}(1-p)^{j-2-k}
$$

(The detailed proof of this inequality is provided in Appendix A.)

Since the algorithm makes $\log j$ decisions to locate the final position of taxon $s_{j+1}$, the probability that the algorithm locates the correct position for taxon $s_{j+1}$ is at least

$$
\left[1-\sum_{k=\frac{j-2}{2}}^{j-2}\left(\begin{array}{c}
j-2 \\
k
\end{array}\right) p^{k}(1-p)^{j-2-k}\right]^{\log j} .
$$

Therefore, the algorithm can construct $T_{\text {true }}$ with a probability at least 


$$
(1-p) \prod_{j=4}^{n-1}\left[1-\sum_{k=\frac{j-2}{2}}^{j-2}\left(\begin{array}{c}
j-2 \\
k
\end{array}\right) p^{k}(1-p)^{j-2-k}\right]^{\log j} .
$$

The first term, $1-p$, is the probability that the algorithm chooses a correct starting quartet topology.

\section{Improvements}

We can see that the maximum probability of algorithm QVOTE to make a wrong decision, $\sum_{k=\frac{j-2}{2}}^{j-2}\left(\begin{array}{c}j-2 \\ k\end{array}\right) p^{k}(1-p)^{j-2-k}$, is close to 0 , when $j$ is relatively large. Therefore, the probability that the algorithm can reconstruct $T_{\text {true }}$ mainly depends on the correctness of the phylogeny with the first several inserted taxa. Based on this observation, we propose the following improvement to algorithm Q-VOTE to look for a good starting phylogeny that contains $m$ taxa for $m \geq 4$.

Given a taxon set $S$, each subset of $m(m \geq 4)$ taxa of $S$ is called an $m$-subset of $S$. A quartet topology is associated with an $m$-subset if the four taxa of the quartet topology are all in the $m$-subset. An $m$-subset is compatible with $Q$ if the set of its associated quartet topologies in $Q$ is compatible. It is easy to see that a compatible $m$-subset has exactly one topology, which can be constructed from its associated quartet topologies in $Q$.

In the following, we only consider $m=5$, while our conclusion can be generalized to larger $m$ with increased running time. The new algorithm, called M-VOTE, first goes through all the possible 5-subsets to find a compatible 5subset. If successful, M-VOTE starts with the phylogeny on the compatible 5-subset and proceeds as Q-VOTE to insert all the other taxa into the phylogeny one by one. If unsuccessful, M-VOTE starts with a randomly selected quartet topology, and it reduces to Q-VOTE. A high-level description of algorithm M-VOTE is summarized in Table 3.

Theorem 4 When $0<p<\frac{1}{3}$ and Step 1 of algorithm M-VOTE is successful, then the algorithm can reconstruct $T_{\text {true }}$ in $O\left(n^{5}\right)$ time with a probability at least

$$
\left[\frac{1}{1+q^{2}+\frac{1}{2} q^{4}+\frac{1}{16} q^{5}}\right] \cdot \prod_{j=5}^{n-1}\left[1-\sum_{k=\frac{j-2}{2}}^{j-2}\left(\begin{array}{c}
j-2 \\
k
\end{array}\right) p^{k}(1-p)^{j-2-k}\right]^{\log j},
$$

where $n$ is the size of the input taxon set, $q=\frac{p}{1-p}$, and $p$ is the quartet error probability of the input quartet topology set.

PROOF. Finding a compatible 5-subset needs $O\left(n^{5}\right)$ time. In each iteration of inserting a taxon into the current phylogeny, the algorithm goes through all the remaining taxa to make a selection. Therefore the overall running time of the algorithm is $O\left(n^{2}+\sum_{i=5}^{n-1} i^{3}(n-i) \log i\right)=O\left(n^{5}+n^{4} \log n\right)=O\left(n^{2}\right)$.

Suppose that in Step 1 the phylogeny constructed from the compatible 5-subset is $T_{5}$ and the true phylogeny of this 5 -subset is $T_{5}^{\prime}$. Note that there are 15 possible phylogenies on this 5 -subset, including $T_{5}^{\prime}$ itself. If $T_{5} \neq T_{5}^{\prime}$, then it is easy to see that $\left|Q_{T_{5}}-Q_{T_{5}^{\prime}}\right|=2,4$, or 5 .

Under the assumption that every quartet topology has probability $p$ to be erroneous, we show in the following that $\left|Q_{T_{5}}-Q_{T_{5}^{\prime}}\right|$ has different probabilities to be $0,2,4$, and 5 (but no probability to be 1 or 3 ).

First of all, clearly, $\left|Q_{T_{5}}-Q_{T_{5}^{\prime}}\right|=0$ as probability $(1-p)^{5}$, since every one of the 5 quartet topologies has to be correct. For each phylogeny $T_{5}$ such that $\left|Q_{T_{5}}-Q_{T_{5}^{\prime}}\right|=2$, i.e., there are two quartet errors, we conclude that these two quartet errors must contain a common subset of three taxa out of the five, and the induced sub-phylogeny of $T_{5}^{\prime}$ on these three taxa should not contain any other taxon from the five. Since the probability to observe $T_{5}$ is $\frac{1}{4} p^{2}(1-p)^{3}$ and there are exactly four possible topologies for $T_{5},\left|Q_{T_{5}}-Q_{T_{5}^{\prime}}\right|=2$ has probability $4 \times$ $\frac{1}{4} p^{2}(1-p)^{3}$. A similar analysis shows that there are eight possible $T_{5}$ 's such that $\left|Q_{T_{5}}-Q_{T_{5}^{\prime}}\right|=4$, and $\left|Q_{T_{5}}-Q_{T_{5}^{\prime}}\right|$ $=4$ has probability $8 \times \frac{1}{16} p^{4}(1-p)$; there are two possible $T_{5}$ 's such that $\left|Q_{T_{5}}-Q_{T_{5}^{\prime}}\right|=5$, and $\left|Q_{T_{5}}-Q_{T_{5}^{\prime}}\right|=5$ has probability $2 \times \frac{1}{32} p^{5}$.

To summarize, the probability of observing incorrect phylogenies on this 5 -subset is 


$$
p^{2}(1-p)^{3}+\frac{1}{2} p^{4}(1-p)+\frac{1}{16} p^{5}
$$

and thus the probability of obtaining a phylogeny $T_{5}$ and $T_{5}=T_{5}^{\prime}$ is

$\frac{(1-p)^{5}}{(1-p)^{5}+p^{2}(1-p)^{3}+\frac{1}{2} p^{4}(1-p)+\frac{1}{16} p^{5}}=\frac{1}{1+q^{2}+\frac{1}{2} q^{4}+\frac{1}{16} q^{5}}$

where $q=\frac{p}{1-p}<\frac{1}{2}$ (and the success probability is greater than 0.779 ) when $0<p<\frac{1}{3}$. After the 5 -subset is identified, M-VOTE proceeds as Q-VOTE and therefore it can construct $T_{\text {true }}$ with a probability at least

$$
\left[\frac{1}{1+q^{2}+\frac{1}{2} q^{4}+\frac{1}{16} q^{5}}\right] \cdot \prod_{j=5}^{n-1}\left[1-\sum_{k=\frac{j-2}{2}}^{j-2}\left(\begin{array}{c}
j-2 \\
k
\end{array}\right) p^{k}(1-p)^{j-2-k}\right]^{\log j} .
$$

Notice that to increase the success probability, Step 1 of algorithm M-VOTE can be changed to search for a compatible $m$-subset for any $m>5$. Furthermore, if the search is not successful, then the algorithm can look for a compatible ( $m-1)$-subset, and so on. In the worst case, the starting phylogeny is a randomly selected quartet topology, which has $1-p$ probability not to be an error. In the following lemma, we show that if the number of quartet errors is not too large or the quartet error probability $p$ is small, then we can almost always find a compatible $m$ subset for $m \geq 5$.

Lemma 5 Given a quartet topology set $Q$ with $k$ quartet errors, there exists at least one compatible $m$-subset if $k<\frac{|Q|}{\left(\begin{array}{c}m \\ 4\end{array}\right)}$, where $m \geq 5$.

PROOF. Given an $m$-subset $\left\{s_{1}, s_{2}, \ldots, s_{m}\right\}$, there are $\left(\begin{array}{c}m \\ 4\end{array}\right)$ quartet topologies in $Q$ that are associated with it. If the set of these $\left(\begin{array}{c}m \\ 4\end{array}\right)$ quartet topologies is not compatible, then there must exist at least one quartet error in it. Since a quartet topology is associated with exactly $\left(\begin{array}{c}n-4 \\ m-4\end{array}\right) m$ subsets, the total number of $m$-subsets associated with at least one quartet error is at most $\left(\begin{array}{c}n-4 \\ m-4\end{array}\right) k<\frac{\left(\begin{array}{c}n-4 \\ m-4\end{array}\right)\left(\begin{array}{l}n \\ 4\end{array}\right)}{\left(\begin{array}{c}m \\ 4\end{array}\right)}=\left(\begin{array}{c}n \\ m\end{array}\right)$. Note that there are $\left(\begin{array}{c}n \\ m\end{array}\right) m$ -

subsets. Therefore, at least one $m$-subset is compatible.

Given a quartet error probability $p$, the expected number of quartet errors in $Q$ is $p|Q|$. It follows from Lemma 5 that if $p<\frac{1}{\left(\begin{array}{c}m \\ 4\end{array}\right)}$, then there is a high probability for the existence of a compatible $m$-subset. For instance, when $p$ $<0.05$, algorithm M-VOTE almost always find a compatible 5-subset (and the probability that the associated phylogeny is correct is at least 0.984; see Figure 2).

\section{Experimental results}

To investigate the practical performance of algorithm $\mathrm{M}$ VOTE, we performed experiments on a set of synthetic data. For a set $S$ of $n$ taxa, we generated a phylogeny by recursively joining randomly selected subtrees. The subtrees were selected from a set that initially only contained the one-node subtrees each corresponding to a given taxon. When two subtrees were joined, we replaced them in the set by the newly generated subtree. The resulting phylogeny on $n$ taxa was treated as the "true" phylogeny $T_{\text {true }}$. A complete quartet topology set, denoted as $Q_{T_{\text {true }}}$, was then induced by this phylogeny. For every quartet on $S$, we altered its topology in $Q_{T_{\text {true }}}$ by a probability $p(0<p$

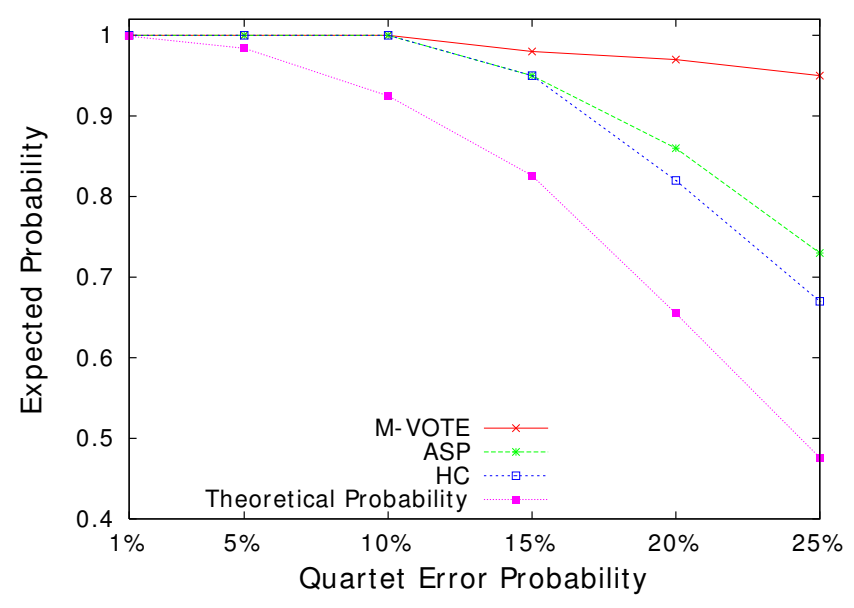

Figure 2

Probability comparison among the proposed algorithm MVOTE, the hypercleaning algorithm ( $\mathrm{HC})$, the answer set programming method for the MQC problem (ASP), and the theoretical success probability of M-VOTE from Theorem 4. 
$<\frac{1}{3}$ ) into a topology randomly selected from the other two possible topologies for the quartet. We treated the altered quartet topologies as quartet errors and the resulting quartet topology set as the input to the algorithms in our experiments. Each generated dataset is labeled by a pair $(n, p)$ where $n$ is the number of taxa and $p$ records the quartet error probability of the input complete quartet topology set. We used the quartet error probability $p=1 \%$, $5 \%, 10 \%, 15 \%, 20 \%, 25 \%$, and the taxon set size $n=20$, $25,30,35,40,45,50$. For every pair of $(n, p)$, we generated 100 datasets. Therefore, given a quartet error probability $p$, we have 700 datasets associated with it. In our experiments, we compared our proposed algorithm M-VOTE with the hypercleaning algorithm (HC) [18], and the answer set programming method (ASP) for the MQC problem [15] in terms of the probability to construct "true" phylogenies.

Given a dataset $D$ and an algorithm $A$, let the phylogeny constructed by algorithm $A$ from $D$ be $T_{D}$ and the "true" phylogeny of $D$ be $T_{\text {true. }}$. If $\left|Q_{L_{D}}-Q_{T_{\text {true }}}\right|=0$, then we say that dataset $D$ can be correctly recovered by algorithm $A$. Given a probability value $p$, we applied each algorithm to the corresponding 700 datasets, and calculated the total number of datasets that could be correctly recovered, referred to as $c$. We then used $\frac{c}{700}$ as the expected probability of the algorithm to construct "true" phylogenies. In our experiments, we used the expected probability as a score to quantify the performance of the algorithms. In Figure 2, we compare the expected probability values of M-VOTE, HC, and ASP, and the theoretical success probability values based on Theorem 4. As shown in Figure 2, algorithm M-VOTE produced "true" phylogenies with the highest probability, and the probability values of algorithm M-VOTE were always higher than the theoretical ones. As the reported time complexity of the hyper-cleaning algorithm $\left(O\left(n^{5} f(2 m)+n^{7} f(m)\right)\right)$ is much higher than that of our algorithm M-VOTE, and the ASP method is an exact method for the NP-hard MQC problem, M-VOTE is therefore the fastest and most accurate one.

\section{Discussion and Conclusions}

In this paper, we have proposed an $O\left(n^{2}\right)$-time algorithm (Q-RAND) to reconstruct a phylogeny from a quartet topology set without quartet errors. This algorithm achieves the optimal lower bound on the number of quartet topology queries. We have also proposed a probabilistic model for the quartet-based phylogeny reconstruction.
Under this model, two algorithms (Q-VOTE and MVOTE) are proposed to reconstruct a phylogeny on a quartet topology set with errors. These two algorithms are mathematically guaranteed to reconstruct the "true" phylogeny with high success probabilities. The key to our algorithms for being able to achieve a high success probability is that for making a wrong decision on the location of a new taxon, there must exist a large number of quartet errors among the queried quartet topologies, which is unlikely. Although we only showed that this is a small probability event under the binomial distribution, we believe that this should be a small probability event also under other probability distributions. The experimental results showed that algorithm M-VOTE produced "true" phylogenies with a higher probability than the theoretical success probability stated in Theorem 4, and it outperformed two existing phylogeny reconstruction methods in both speed and accuracy.

This work opens up several research directions. First of all, in real world phylogeny reconstruction, the distribution of quartet errors is largely unknown, both theoretically and empirically. The probabilistic model and algorithms proposed in this paper can be regarded as the first step toward reconstructing the "true" phylogeny with a high success probability. Csürös and Kao [1] proposed an algorithm that can reconstruct the true phylogeny with a high probability in the Jukes-Cantor model of evolution [23]. Our next step would be to investigate possible probabilistic properties of the quartet topology set under some models of evolution and to design algorithms that can reconstruct the true phylogeny with a high probability under such evolutionary models. Secondly, it would be interesting to investigate the relationships between the accuracy of the reconstructed phylogeny and the topology of the true phylogeny. In general, the larger the quartet error probability $p$ is, the more difficult it is to reconstruct the true phylogeny and therefore the lower the accuracy is. However, under the same quartet error probability, it is interesting to investigate whether different topologies of the true phylogeny may affect the accuracy of our algorithms. Thirdly, some computational questions are still open. Can we reduce the running time of the proposed algorithms by utilizing the techniques proposed in [2022]? We know that there is a trade-off between the running time and the number of queried quartet topologies, as demonstrated in Theorem 4 . If we attempt to reduce the running time by querying fewer quartet topologies, what is the success probability of the new algorithm to reconstruct the true phylogeny?

\section{Appendix A}

Theorem 6 If $N$ is an even number and $0<p<\frac{1}{3}$, then 


$$
\sum_{k=\frac{N}{2}}^{N}\left(\begin{array}{l}
N \\
k
\end{array}\right) p^{k}(1-p)^{N-k} \geq \sum_{k=\frac{N}{2}+1}^{N+1}\left(\begin{array}{c}
N+1 \\
k
\end{array}\right) p^{k}(1-p)^{N+1-k}
$$

and

$$
\sum_{k=\frac{N}{2}}^{N}\left(\begin{array}{c}
N \\
k
\end{array}\right) p^{k}(1-p)^{N-k} \geq \sum_{k=\frac{N}{2}+1}^{N+2}\left(\begin{array}{c}
N+2 \\
k
\end{array}\right) p^{k}(1-p)^{N+2-k}
$$

PROOF. For the first inequality,

$$
\begin{aligned}
\frac{k+1}{N+1} \geq p & \Leftrightarrow\left(\begin{array}{c}
N \\
k
\end{array}\right) \geq\left(\begin{array}{c}
N+1 \\
k+1
\end{array}\right) p \\
& \Leftrightarrow\left(\begin{array}{c}
N \\
k
\end{array}\right) p^{k}(1-p)^{N-k} \geq\left(\begin{array}{c}
N+1 \\
k+1
\end{array}\right) p^{k+1}(1-p)^{N-k} \\
& \Leftrightarrow \sum_{k=\frac{N}{2}}^{N}\left(\begin{array}{l}
N \\
k
\end{array}\right) p^{k}(1-p)^{N-k} \geq \sum_{k=\frac{N}{2}+1}^{N+1}\left(\begin{array}{c}
N+1 \\
k
\end{array}\right) p^{k}(1-p)^{N+1-k} .
\end{aligned}
$$

For the second inequality, it is easy to prove that

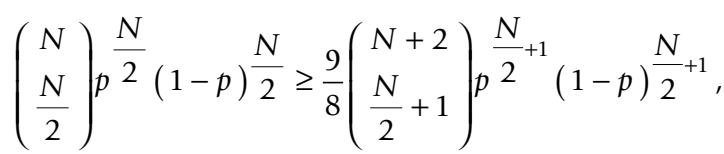

$$
\begin{aligned}
& \left(\begin{array}{c}
N \\
\frac{N}{2}+1
\end{array}\right) p^{\frac{N}{2}+1}(1-p) \frac{N}{2}-1 \geq\left(\begin{array}{c}
N+2 \\
\frac{N}{2}+2
\end{array}\right) p^{\frac{N}{2}+2}(1-p) \frac{N}{2} \\
& \left(\begin{array}{c}
N \\
\frac{N}{2}+2
\end{array}\right) p^{\frac{N}{2}+2}(1-p) \frac{N}{2}-2 \geq\left(\begin{array}{l}
N+2 \\
\frac{N}{2}+3
\end{array}\right) p^{\frac{N}{2}+3}(1-p)^{\frac{N}{2}-1} \text {, } \\
& \frac{1}{8}\left(\begin{array}{l}
N+2 \\
\frac{N}{2}+1
\end{array}\right) p^{\frac{N}{2}+1}(1-p) \frac{N}{2}+1 \geq\left(\begin{array}{l}
N+2 \\
\frac{N}{2}+4
\end{array}\right) p^{\frac{N}{2}+4}(1-p) \frac{N}{2}-2 \\
& \left(\begin{array}{c}
N \\
\frac{N}{2}+3
\end{array}\right) p^{\frac{N}{2}+3}(1-p)^{\frac{N}{2}-3} \geq\left(\begin{array}{c}
N+2 \\
\frac{N}{2}+5
\end{array}\right) p^{\frac{N}{2}+5}(1-p)^{\frac{N}{2}-3} \\
& p^{N} \geq p^{N+2} \text {. }
\end{aligned}
$$

Therefore,

$$
\sum_{k=\frac{N}{2}}^{N}\left(\begin{array}{c}
N \\
k
\end{array}\right) p^{k}(1-p)^{N-k} \geq \sum_{k=\frac{N}{2}+1}^{N+2}\left(\begin{array}{c}
N+2 \\
k
\end{array}\right) p^{k}(1-p)^{N+2-k}
$$

\section{Authors' contributions}

All authors contributed equally to this work, and read and approved the final manuscript.

\section{Acknowledgements}

The research of GW, GL, and JHY is partially supported by NSERC. GL is also supported by CFI. JHY is also supported by NSFC 60673009 . We thank the anonymous reviewers for their extremely helpful comments.

\section{References}

I. Csürös M, Kao MY: Provably fast and accurate recovery of evolutionary trees through harmonic greedy triplets. SIAM Journal on Computing 200I, 31 :306-322.

2. Saitou N, Nei M: The neighbor-joining method: a new method for reconstructing phylogenetic trees. Molecular Biology and Evolution 1987, 4:406-425.

3. Moret BME, Wang LS, Warnow T: Toward new software for computational phylogenetics. IEEE Computer 2002, 35(7):55-64.

4. Pelleg D: Algorithms for constructing phylogenies from quartets. In Master's thesis Israel Institute of Technology; 1998.

5. Ben-Dor A, Chor B, Graur D, Ophir R, Pelleg D: From four-taxon trees to phylogenies (preliminary report): The Case of Mammalian Evolution. Proceedings of the 2nd Annual International Conference on Computational Molecular Biology 1998:9-19.

6. Fitch WM, Margoliash E: Construction of phylogenetic trees. Science 1967, 155:279-284.

7. Kearney PE: The ordinal quartet method. Proceedings of the 2 nd Annual International Conference on Computational Molecular Biology 1998:125-134

8. Erdős PL, Steel M, Székély L, Warnow T: Constructing big trees from short sequences. In Lecture Notes in Computer Science I256: Proceedings of the 24th International Colloquium on Automata, Languages, and Programming Edited by: Goos G, Hartmanis J, van Leeuwen J. New York, NY: Springer-Verlag; 1997:827-837.

9. Erdős PL, Steel MA, Székely LA, Warnow T: A few logs suffice to build (almost) all trees I. Random Structures and Algorithms I997, 14:153-184.

10. Strimmer K, von Haeseler A: Quartet puzzling: a quartet maximum-likelihood method for reconstructing tree topologies. Molecular Biology and Evolution 1996, I3(7):964-969.

II. Davison AC, Hinkley DV:: Bootstrap Methods and Their Applications Cambridge, U.K.: Cambridge University Press; 1997.

12. Swofford DL, Olsen GJ, Waddell PJ, Hillis DM: Phylogenetic Inference. In Molecular Systematics 2nd edition. Edited by: Hillis DM, Moritz C, Mable BK. Sunderland, MA: Sinauer Associates; 1996:407-5।4.

13. Jiang T, Kearney P, Li M: Some open problems in computational molecular biology. Journal of Algorithms 2000, 34:194-201.

14. Gramm J, Niedermeier R: A fixed-parameter algorithm for minimum quartet inconsistency. Journal of Computer and System Sciences 2003, 67:723-74I.

15. Wu G, Lin G, You J: Quartet based phylogeny reconstruction with answer set programming. Proceedings of the I 6 th IEEE International Conference on Tools with Artificial Intelligence 2004:612-6I9.

16. Jiang T, Kearney P, Li M: A polynomial time approximation scheme for inferring evolutionary trees from quartet topologies and its application. SIAM Journal on Computing 2000, 30: $1942-196 \mid$.

17. Vedova GD, Jiang T, Li J, Wen J: Approximating minimum quartet inconsistency (abstract). Proceedings of the 13th Annual ACMSIAM Symposium on Discrete Algorithms 2002:894-895.

18. Berry V, Bryant D, Jiang T, Kearney P, Li M, Wareham T, Zhang H: A practical algorithm for recovering the best supported edges of an evolutionary tree (extended abstract). Proceedings of the IIth Annual ACM-SIAM Symposium on Discrete Algorithms 2000:287-296.

19. Jordan C: Sur les assemblages de lignes. Journal für die Reine und Angewandte Mathematik 1869, 70:185-190.

20. Kannan SK, Lawler EL, Warnow T: Determining the evolutionary tree using experiments. Journal of Algorithms 1996, 21:26-50.

21. Kao MY, Lingas A, Östlin A: Balanced randomized tree splitting with applications to evolutionary tree constructions. In Lecture Notes in Computer Science 1563: Proceedings of the 16th Interna- 
tional Symposium on Theoretical Aspects of Computer Science Edited by: Meinel C, Tison S. New York, NY: Springer-Verlag; 1999:184-196.

22. Brodal GS, Fagerberg R, Pedersen CNS, Östlin A: The complexity of constructing evolutionary trees using experiments. In Lecture Notes in Computer Science 2076: Proceedings of the 28th International Colloquium on Automata, Languages, and Programming Edited by: Orejas F, Spirakis PG, van Leeuwen J. New York, NY: Springer-Verlag; 2001:140-I5I.

23. Jukes TH, Cantor CR: Evolution of protein molecules. In Mammalian Protein Metabolism Volume III. Edited by: Munro HN. New York, NY: Academic Press; 1969:21-132.

Publish with Biomed Central and every scientist can read your work free of charge

"BioMed Central will be the most significant development for disseminating the results of biomedical research in our lifetime. " Sir Paul Nurse, Cancer Research UK

Your research papers will be:

- available free of charge to the entire biomedical community

- peer reviewed and published immediately upon acceptance

- cited in PubMed and archived on PubMed Central

- yours - you keep the copyright

Submit your manuscript here:

http://www.biomedcentral.com/info/publishing_adv.asp
BioMedcentral 